\title{
Recursos tecnológicos de apoio ao ensino na saúde
}

\section{Technological resources to support education in health}

\author{
Cristiane Martins Peres ${ }^{1}$, Kátia Mitiko Firmino Suzuki², Paulo Mazzoncini de Azevedo-Marques ${ }^{3}$
}

\begin{abstract}
RESUMO
A inserção das Tecnologias da Informação e Comunicação (TICs) no universo da atenção à saúde tem conduzido a mudanças de métodos de ensino e de processos na assistência, gerando também novos conceitos, com destaque para o de eSaúde. No início do século XXI um conjunto de inciativas para apoio ao uso das TICs nos diversos contextos da área da saúde passa a ganhar corpo no Brasil, tendo como destaques, o Telessaúde Brasil Redes, UNA-SUS (Universidade Aberta do SUS) e RUTE (Rede Universitária de Telemedicina). O foco desses programas se concentra no aperfeiçoamento contínuo dos profissionais com finalidades de atualização, capacitação técnica, cursos de aperfeiçoamento e discussão de casos clínicos na área da saúde. O movimento da utilização das TICs também tem progredido em direção a melhoria do processo de ensino e aprendizagem nos cursos de graduação, contudo ainda de forma limitada. Os recursos online e modelos de EAD que podem ser utilizados são muitos. Mas, ainda que várias instituições os ofereçam, há todo um entrono de planejamento educacional que precisa ser considerado. O papel da docência nesse contexto deve ser bem definido, para que as competências específicas para potencializar possíveis sucessos na utilização das TICs sejam desenvolvidas pelos docentes. Em uma análise com perspectiva institucional, evidencia-se a necessidade de estruturação de núcleos multiprofissionais como forma de apoio ao estudo, modelagem e implantação de estratégias e de programas que promovam uma utilização mais efetiva das TICs no cenário das ciências da saúde.
\end{abstract}

Palavras-chave: Tecnologia da Informação. Tecnologias da Informação e Comunicação. Educação a Distância. Educação em Saúde, Telessaúde.

\begin{abstract}
The integration of Information and Communication Technologies (ICTs) on the universe of health care has led to changes in teaching methods and processes in care, also generating new concepts, highlighting the eHealth. At the beginning of the XXI century a number of initiatives to support the use of ICTs in different contexts of health begins to take shape in Brazil, with the highlights, the Brazil Tele health Networks, UNA-SUS (SUS Open University) and RUTE (University Telemedicine Network). The focus of these programs are on continuous professional education, technical training, training courses and discussion of clinical cases in healthcare. The movement of the use of ICT has also made progress toward improving
\end{abstract}

1. Pedagoga e Psicopedagoga do Centro de Apoio Educacional e Psicológico da Faculdade de Medicina de Ribeirão - USP.

2. Analista de Sistemas, Chefe da Seção Técnica de Informática Faculdade de Medicina de Ribeirão - USP.

3. Professor Associado em Bioinformática e Física Medica junto ao Centro de Ciências das Imagens e Física Médica - Departamento de Clínica Médica da Faculdade de Medicina de Ribeirão - USP.
Correspondência: Avenida dos Bandeirantes 3.900, Rua das Paineiras, casa 08. Centro de Apoio Educacional e Psicológico da FMRP-USP CEP 14049-900 - Ribeirão Preto, São Paulo, Brasil.

Artigo recebido em 25/11/2014 Aprovado para publicação em 18/12/2014 
the teaching and learning process in undergraduate courses, however still been limited. Online resources and EAD models that can be used are many. But there are important educational planning necessities that needs to be considered. The role of teaching in that context must be clearly defined so that specific skills to enhance success potential in the use of ICTs can be developed by teachers. In an analysis with institutional perspective, the need for the structuring of multi-professional center is highlighted as a way to support the study, modeling and implementation of strategies and programs that promote more effective use of ICTs in the health sciences setting.

Key words: Information Technology. Information and Communication Technologies. Distance Education. Health Education. Telehealth.

\section{Introdução}

\section{Tecnologia da informação e tecnologias da informação e comunicação}

O termo Tecnologia da Informação (TI) serve para designar o conjunto de recursos tecnológicos e computacionais para a geração e uso da informação, fundamentada em componentes como: hardware (dispositivos e periféricos), software e seus recursos, sistemas de telecomunicações e gestão de dados e informações. Uma vez que se vivencia na "era da informação", a tecnologia da informação tornou-se uma parte natural da vida cotidiana. ${ }^{1} \mathrm{O}$ termo Tecnologias $d a$ Informação e Comunicação (TICs) refere-se ao conjunto de tecnologias que permitem o acesso à informação através do uso de telecomunicação. É semelhante à Tecnologia da Informação (TI), mas concentra-se principalmente em tecnologias de comunicação. Telecomunicação, por sua vez, é a transmissão de sinais a longas distâncias, por exemplo através da Internet e das redes de telefonia celular.

O uso das TICs vem transformando o cotidiano das atividades humanas, sendo que, na década de 1970 o foco esteve sobre os serviços bancários, na década de 1980 sobre os processos industriais; e, desde o final da década de 1990 e início da década de 2000 a atenção voltou-se principalmente para a área da Saúde. A inserção da TI e das TICs no universo da atenção à saúde tem conduzido a mudanças de métodos e processos, gerando também alguns conceitos novos, com destaque para o de eSaúde, do Inglês eHealth.

eSaúde pode ser definida como um modelo de atenção integral e integrada à saúde, baseada em Tecnologias da Informação e Comunicação (TICs) e dispositivos eletrônicos, incluindo sistemas de informação em saúde, prontuário eletrônico do paciente, prescrição eletrônica, saúde móvel, telemedicina e telessaúde, entre outros. ${ }^{2}$ Em tese, a adoção de um modelo de eSaúde poderia melhorar o acompanhamento, o gerenciamento, e a qualidade da atenção à saúde do indivíduo e de sua família. Poderia também auxiliar a redução de ineficiências na atenção à saúde, ampliar o acesso, reduzir custos, aumentar a qualidade e propiciar uma medicina mais personalizada e precisa.

\section{Tecnologias da informação e comunicação no ensino na saúde}

A partir da década de 1990, percebe-se um aumento significativo no número de estudos sobre a educação mediada por tecnologias na área da saúde e suas diversas ferramentas e recursos. ${ }^{3}$ Mais recentemente, a partir da segunda metade da década de 2000, o uso das TICs para suporte a atividades de atenção e de ensino na saúde no Brasil tem sido bastante ampliado, principalmente devido à criação de programas governamentais voltados para a implantação, disseminação e fortalecimento de um modelo nacional de eSaúde, com destaque para o Telessaúde Brasil Redes, UNA-SUS (Universidade Aberta do SUS) e RUTE (Rede Universitária de Telemedicina).

O Programa Nacional de Telessaúde, agora chamado Telessaúde Brasil Redes, foi instituído no âmbito do Ministério da Saúde pela Portaria Ministerial $\mathrm{n}^{\mathrm{o}} 35$ de 04 de janeiro de 2007, com o objetivo de desenvolver ações de apoio à assistência à saúde e, sobretudo, de educação permanente na Estratégia de Saúde da Família, visando a educação para o trabalho, com perspectiva de mudanças de práticas que resultem na melhoria da qualidade do atendimento da Atenção Básica no SUS. A implantação do Programa Nacional de Telessaúde se efetivou a partir de uma ampla ação governamental inter setorial coordenada pelo Ministério da Saúde, por meio da Secretaria de Gestão do Trabalho e da Educação na Saúde (SGTES) e Secretaria de Atenção à Saúde (SAS), com a participação, do Ministério da Educação, por meio das Secretarias de Educação Superior (SESu) e de Educação à Distância (SEED), Casa Civil, da Organização Pan-Americana da Saúde, dos Ministérios da 
Ciência, Tecnologia e Inovação, da Defesa e Ministério das Comunicações, além de várias universidades públicas e entidades como a BIREME, o Conselho Federal de Medicina e a Sociedade Brasileira de Medicina de Família e Comunidade. O objetivo do Telessaúde Brasil Redes é integrar as equipes de saúde da família das diversas regiões do país com os centros universitários de referência, para melhorar a qualidade dos serviços prestados em atenção primária, diminuindo o custo de saúde através da qualificação profissional, redução da quantidade de deslocamentos desnecessários de pacientes e aumento de atividades de prevenção de doenças. ${ }^{4}$

De forma independente, porém com escopo de atuação evidentemente complementar ao Telessaúde Brasil Redes, foi inaugurada em $1^{\circ}$ de agosto de 2007 a Rede Universitária de Telemedicina (RUTE). A RUTE é uma iniciativa do Ministério da Ciência, Tecnologia e Inovação, apoiada pela Financiadora de Estudos e Projetos (Finep) e pela Associação Brasileira de Hospitais Universitários (Abrahue), sob a coordenação da Rede Nacional de Ensino e Pesquisa (RNP), que visa a apoiar o aprimoramento da infraestrutura para telemedicina em hospitais universitários, bem como promover a integração de projetos entre as instituições participantes. Para tanto, a RUTE propõe-se a prover a infraestrutura de serviços de comunicação, assim como parte dos equipamentos de informática e comunicação para os grupos de pesquisa, promovendo integração e conectividade e disseminando atividades de $\mathrm{P} \& \mathrm{D}$ (Pesquisa \& Desenvolvimento) das instituições participantes, operacionalizando grupos multi-institucionais de discussão, chamados SIGs (Special Interest Groups). 5,6,7

Ainda dentro do mesmo conjunto de inciativas para apoio ao uso das TICs na atenção e no ensino na saúde, em 18 de junho de 2008 foi criada a Universidade Aberta do SUS (UNA-SUS), cujo objetivo é criar condições para o funcionamento de uma rede colaborativa de instituições acadêmicas, serviços de saúde e gestão do SUS, destinada a atender necessidades de formação e educação permanente em saúde em áreas estratégicas. Essa rede funciona por meio do intercâmbio de experiências, cooperação para desenvolvimento e implementação de ações educacionais mediadas por tecnologias interativas, compartilhamento de recursos educacionais, apoio presencial ao processo de aprendizagem em serviço e bolsas de educação pelo trabalho em saúde (Lei 11.129/2005) para tutoria inter-pares.
A UNA-SUS apoia o funcionamento dessa rede através do oferecimento de dois serviços: o Acervo de Recursos Educacionais em Saúde (ARES) e a Plataforma Arouca. O ARES é o repositório digital da UNA-SUS aonde são armazenados e ficam disponíveis para consulta os recursos educacionais utilizados pelas instituições que compõem a Rede em suas ofertas de cursos. É um acervo público, com materiais em diversos formatos, alimentado de forma colaborativa e de acesso livre pela internet. A Plataforma Arouca é o Sistema de Informação dos Profissionais de Saúde do Brasil. Nela é possível acessar um mapa de oportunidades educacionais, sejam de curta ou longa duração, de caráter mais prático ou acadêmico, presenciais ou a distância que são financiadas com recursos públicos para qualificar os trabalhadores do SUS. Assim, todas as ações de educação em saúde em um dado território passam a ser transparentes, possibilitando uma melhor gestão dos recursos investidos em qualificação e maior controle social. Além disso, cada trabalhador de saúde terá, através da plataforma, acesso a um histórico unificado com toda sua trajetória de educação permanente em saúde.

As aplicações da EAD nas ciências da saúde têm ocorrido principalmente no aperfeiçoamento contínuo dos profissionais da saúde com finalidades de atualização, capacitação técnica, discussão de casos clínicos e para o oferecimento de cursos de pós-graduação. Mas, os modelos e estratégias que estão sendo utilizados podem também ser aplicados na graduação, sendo as principais ${ }^{8}$ :

- Leitura dirigida - Possibilita o acompanhamento periódico da literatura cientifica mais relevante seguido de pequenas avalições sobre o material da leitura.

- Desafio Clínico - O aluno é convidado a resolver um caso real e a enviar a justificativa sobre sua solução para um professor pré-determinado.

- Discussão de casos - Pode ser realizada de forma síncrona, por meio do recurso do bate-papo online, videoconferência ou webconferência; ou de forma assíncrona, por meio de ferramentas de fórum e uso de e-mail. Podem ser incluídas informações complementares, como imagens radiográficas e outros tipos de resultados de exames.

- Simulação - É possível se encontrar tanto simulações online de situações clinicas, quanto conteúdos relacionados às áreas básicas e de fundamentos da saúde. Elas contam com roteiros que levam o aluno a evoluir gradativamente. 
- Cursos Modulares - O foco desse modelo é oferecer temas específicos em um formato assíncrono ou misto, com a finalidade de atualização dos profissionais de saúde. Os recursos utilizados nas plataformas são variáveis.

\section{Educação a Distância}

\section{As gerações tecnológicas}

Os avanços dos recursos de tecnologia na educação evoluíram passando por gerações na história humana. Sob o nome genérico de Educação a Distância, cada época pode ser caracterizada pelo tipo de material instrucional e pelo meio de comunicação utilizados.

A primeira geração foi a do material impresso, tendo como exemplo o ensino por correspondência no início século XIX (no Brasil, em 1939) e a segunda geração, a dos telecursos ou programas que envolviam meios como rádio, TV e material impresso. A comunicação se dava de forma síncrona e sem interação. A terceira geração corresponde, para os autores, ao uso dos recursos da internet, sendo baseada na difusão dos computadores pessoais e nas primeiras tentativas de criação de redes de pesquisa, as quais, sendo completadas com os avanços das telecomunicações, permitiram a eliminação da relação espaço- temporal, possibilitando o acesso à educação assíncrona. Quando ocorre a popularização da banda larga de comunicação, internet, interação por vídeo e ao vivo como as teleconferências e outros recursos interativos em ambientes virtuais, ou seja, ocorrem as primeiras interações em tempo real, tem-se o início da quarta geração, marcada definitivamente pela interatividade. E, finalmente, é na quinta geração da $\mathrm{EAD}$, que os ambientes virtuais de aprendizagem (AVA), passaram a ser incorporados na Educação. ${ }^{9}$

No Brasil, a educação a distância foi reconhecida oficialmente na década de 1990 , e recebe a seguinte definiç̧ão no portal do Ministério da Educação:

"Educação a distância é a modalidade educacional na qual alunos e professores estão separados, física ou temporalmente e, por isso, faz-se necessária a utilização de meios e tecnologias de informação e comunicação. Essa modalidade é regulada por uma legislação específica e pode ser implantada na educação básica (educação de jovens e adultos, educação profissional técnica de nível médio) e na educação superior."
Atualmente, o órgão define as modalidades de educação mediada por tecnologias como:

- Educação a distância ou aprendizado eletrônico (denominado na língua inglesa como e-learning), na qual alunos e professores não estão fisicamente presentes em um ambiente presencial de ensinoaprendizagem. O processo educacional poderá ocorrer independente dos fatores tempo e/ou espaço.

- Ensino semipresencial ou ensino híbrido (denominado na língua inglesa como bimodal education ou blended learning), que combina estudos presenciais e a distância. Entretanto, pode-se dizer que nessa modalidade coexistem dois modelos. Sendo que, em um modelo os recursos das tecnologias da informação servem como ferramentas auxiliares ao processo de ensino e aprendizagem presencial e, no outro, os encontros presenciais são mantidos para a realização de provas e em alguns casos para revisões e orientações, com as demais atividades sendo realizadas à distância.

Outras possibilidades como a aprendizagem móvel (conhecida na língua inglesa como m-learning de mobile learning), que está relacionada ao aprendizado por interações que são proporcionadas por meio de dispositivos móveis como celulares, smartphones, ipods e tablets também já surge como aspirante a uma nova modalidade de educação mediada por tecnologia. Sejam os dispositivos móveis, softwares, podcasts ou outros que passem a receber maior status na literatura, os mesmo se enquadram melhor na definição de recursos de apoio as modalidades de educação. $\mathrm{Na}$ medicina esses dispositivos já assumiram papel importante, tanto na prática em saúde, quanto como objeto de estudo em trabalhos da literatura. O ganho principal do uso desses dispositivos é a possibilidade de conectividade a qualquer tempo e em qualquer lugar (que pode ser chamada de ubiquidade).

\section{Modelos de EAD}

Nas experiências pioneiras de educação a distância no Brasil, o público-alvo eram as pessoas sem possibilidade de escolarização, o que gerou certo preconceito com esta modalidade de ensino. Porém, aos poucos, o ensino à distância foi entendido como uma possibilidade de maior amplitude de escolhas para o aluno quanto aos conteúdos e aos meios de aprendizagem. ${ }^{10}$ Atualmente incorpora o entendimento de ser um meio para a atualização contínua daqueles que 
antes, por questões geográficas ou econômicas, se mantinham a margem das novas possibilidades de aprendizagem nas suas áreas de atuação. Pode ser também uma ferramenta de apoio para se alcançar um diploma de ensino superior, principalmente em cursos da área de humanas.

Até hoje mantem-se sob discussão a proposta do modelo de aulas gravadas, usualmente encontrado nos cursos à distância. Pois, estimular o interesse do aluno e descobrir qual a melhor forma de gravar uma aula, que será vista de forma solitária pelo aluno, são, ainda, desafios para o sucesso desse modelo.

O desenvolvimento da EAD no Brasil, de 1994 até hoje, pode ser descrito a partir dos seguintes modelos ${ }^{11}$

1. O modelo de tele-educação, que segue as mesmas características que o de tele-aulas, destacando a transmissão da ação para os polos presenciais. $\mathrm{O}$ papel do professor é transmitir suas aulas ao vivo para alunos que se encontram reunidos em salas presenciais e sob supervisão de um professor tutor local.

2. O modelo de vídeo educação ou modelo vídeo aulas, que destaca a possibilidade do aluno assistir as aulas gravadas em momentos diferentes da aula presencial. Elas podem ser encaminhadas aos alunos em CDs ou DVDs, junto com materiais impressos ou disponibilizadas na web.

3. O modelo de universidade virtual, caracterizado pelo uso intensivo de tecnologias digitais para a entrega de conteúdos e para o apoio a atividades para os alunos, bem como para promover a interação destes com professores, colegas e com o suporte técnico e administrativo. Neste modelo as etapas presenciais são reservadas para a realização de provas, com as demais atividades sendo realizadas a distância.

4. O modelo misto ou semipresencial, em que os alunos dos cursos a distância permanecem períodos regulares na instituição (de forma presencial), aonde realizam não apenas provas, mas atividades em outros ambientes, como por exemplo, laboratórios. Nesse modelo, os ambientes virtuais passam a ser utilizados para gerenciamento de atividades educacionais individuais, coletivas e colaborativas.

Na busca de precisão para o conceito de Educação a Distância, Kenski (2013) afirma que essa modalidade pode ser mais diversificada e complexa do que a forma pela qual o senso comum a compreende. A autora considera que é pre ciso sempre vincular o conceito ao suporte tecnológico prevalente de comunicação e interação (impresso, rádio, televisão, computadores, internet), em um determinado contexto histórico cultural.

\section{Uso dos recursos tecnológicos no ensino na FMRP}

Os recursos tecnológicos disponíveis para o ensino são bastante diversificados, destacando-se alguns como fórum, bibliotecas virtuais, tira-dúvidas, arquivos para download, mural de avisos, bate-papo, recepção e envio de materiais e gerenciadores de tarefas. Esses recursos estão disponíveis na Faculdade de Medicina de Ribeirão Preto (FMRP), para uso no ensino de graduação, pós-graduação e cultura extensão, através da plataforma de aprendizagem Moodle, que é um AVA (Ambiente Virtual de Aprendizagem). Esta plataforma permite que os alunos estudem e interajam utilizando os recursos tecnológicos do ambiente, tais como vídeo-aulas, arquivos para download, tarefas off-line, chat, realização de webconferências, entre outros etc.

As figuras 1 e 2 ilustram o AVA organizado para alguns dos cursos de graduação da FMRP.

Em geral, a estrutura do AVA segue a do modelo curricular do curso, ou seja, está dividida em períodos ou semestres, seguida das disciplinas que compõem cada período. O conteúdo da disciplina é organizado pelo professor, de acordo com as atividades previstas respeitando o seu estilo. Na maioria das disciplinas o professor, inicialmente, relaciona os demais professores que participam da disciplina, tendo em seguida a apresentação do curso, seu calendário de atividades, textos para a leitura complementar (arquivos em formato pdf), vídeo-aulas, questionários e, em alguns casos, também a avaliação é disponibilizada no AVA.

É importante ressaltar que uso dos recursos tecnológicos na EAD, baseado no modelo misto, permite ao professor promover a discussão em sala de aula, ouvir sugestões, propor atividades atualizadas relacionadas ao conteúdo abordado e, assim, aprimorar as modalidades existentes para a construção do conhecimento, em virtude da facilidade para estimular a autonomia dos alunos na construção do seu conhecimento, através de feedback dos comentários/sugestões e das atividades desenvolvidas. 


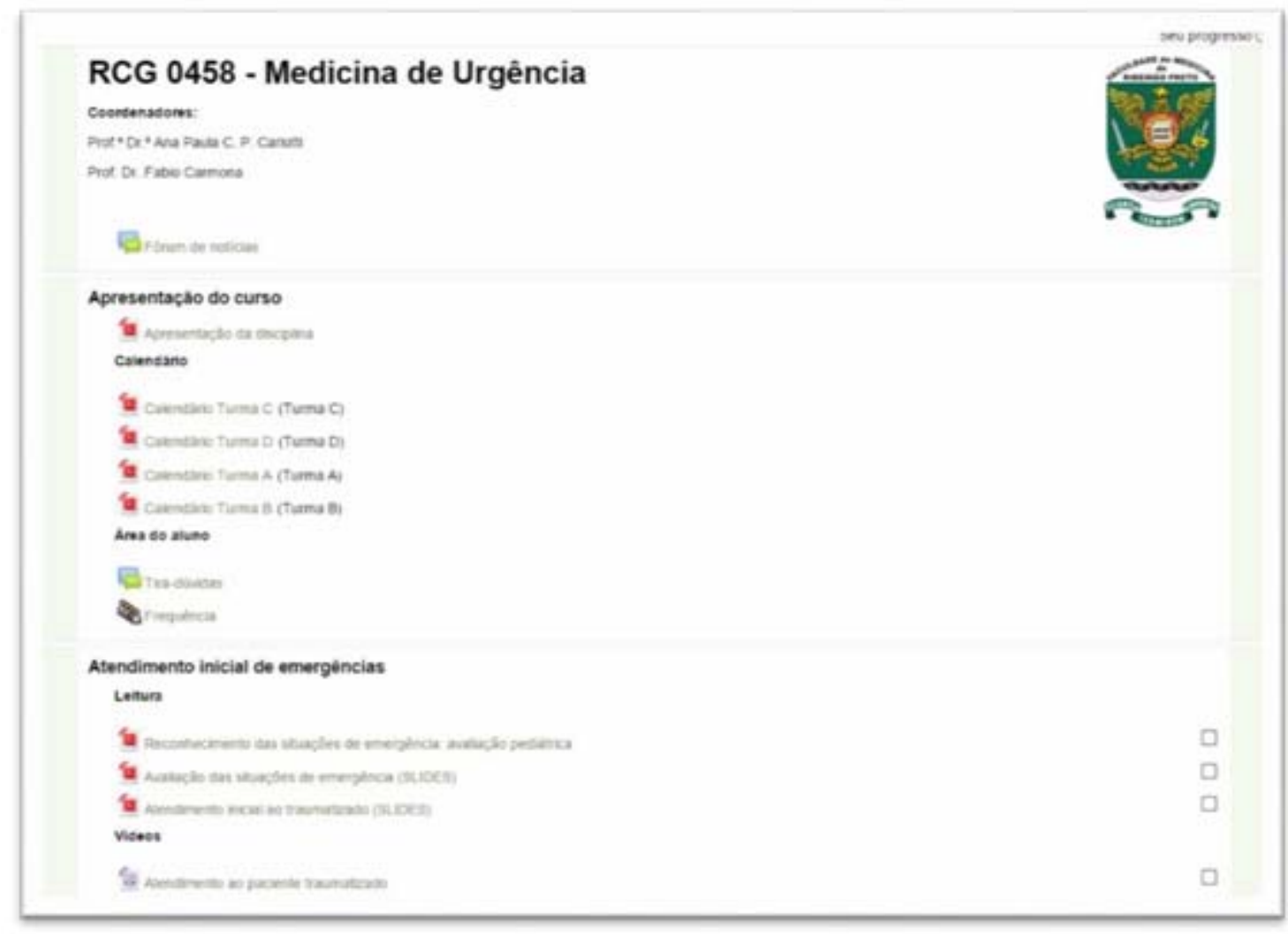

Figura 1: AVA do curso de Medicina - disciplina RCG0458 Medicina de Urgência.

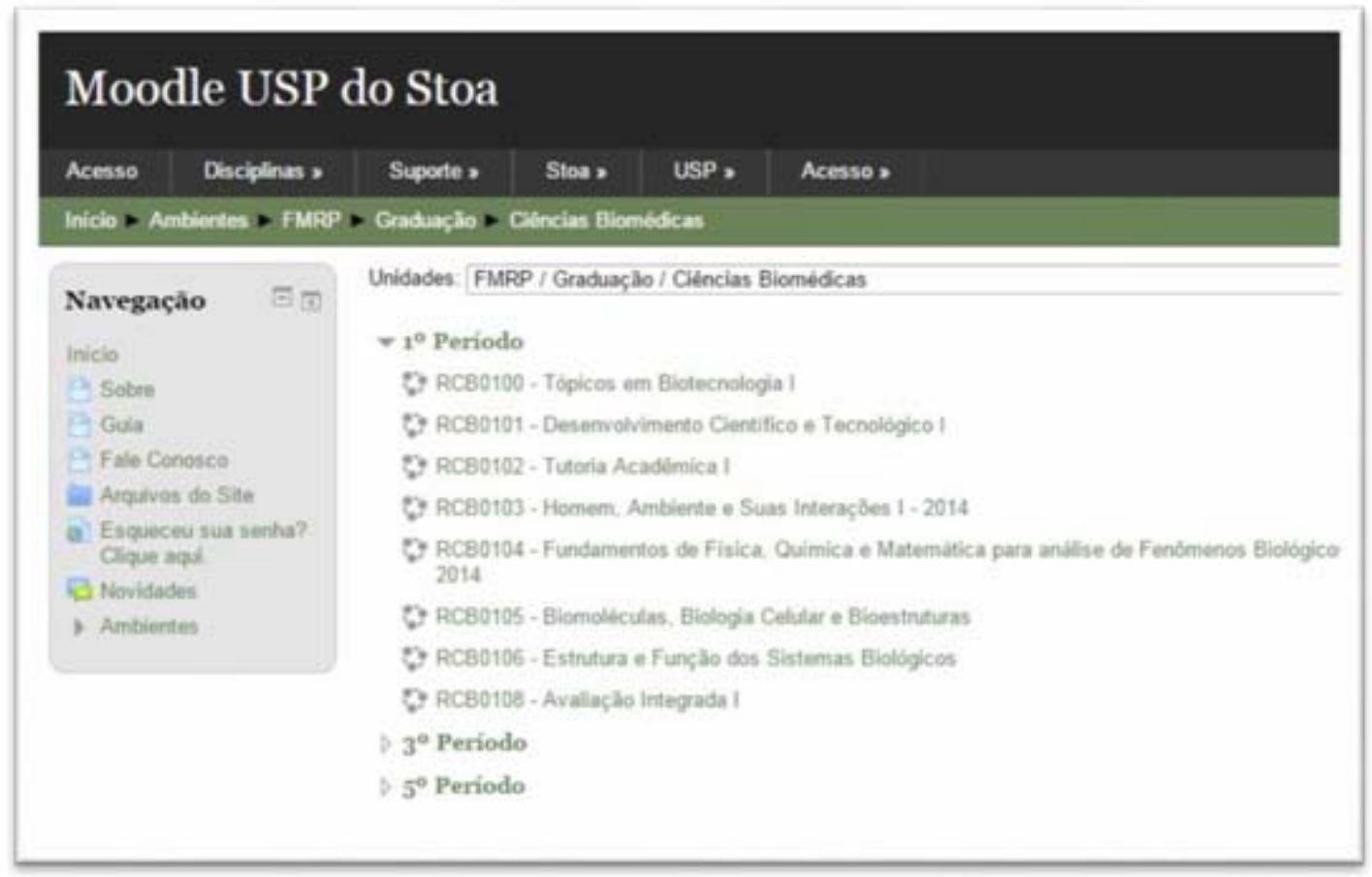

Figura 2: AVA do curso de graduação de Ciências Biológicas da FMRP. 
A figura 3 mostra um exemplo de curso de capacitação de Cultura e Extensão, exibindo uma tela do ambiente para Tutoria Acadêmica-Cientifica e Programa de Desenvolvimento Docente para avaliação de habilidades clínicas.

Outro recurso tecnológico que pode ser utilizado para promover o ensino em EAD na Saúde, principalmente, para viabilizar a transmissão de imagens e áudio de alta definição é o Digital Video Transport System (DVTS). O DVTS é um sistema para a comunicação em tempo real de imagens e de áudio em alta resolução baseada em IP (Internet Protocol) e é gratuito para uso acadêmico e disponível para a maioria das plataformas de sistemas operacionais, tais como: MS Windows, MacOS e GNU/Linux. O DVTS exige poucos recursos de hardware: um microcomputador pessoal acoplado a uma câmera de vídeo através de uma conexão Firewire - IEEE 1394. Entretanto, necessita de banda de conexão com a Internet da ordem de 30 a 35 Mbps só para o envio, e de aproximadamente 70 Mbps para o envio e a recepção simultâneos dos streams (fluxos de dados) de vídeo digital. A transmissão de imagens médicas por DVTS pode ser considerada uma ferramenta de Telemedicina para apoio ao ensino 12. Em 2010, a FMRP testou e aprovou essa tecnologia para transmissão e gravação em tempo real de procedimentos cirúrgicos experimentais.

\section{Considerações Finais}

Embora os modelos de ensino baseados nas TICs possam servir como base para uma parceria bidirecional de aprendizagem entre alunos e professores e entre profissionais da saúde, já se tem bem definido que algumas competências docentes específicas se fazem necessárias para potencializar possíveis sucessos na utilização das TICs. Dentre estas destacamse:

- Capacidade de criar vínculos, mesmo à distância, com os alunos.

- Ter domínio dos recursos tecnológicos disponíveis para que se crie uma rede de comunicação e para que o aprendizado seja multidirecional.

- Adquirir as habilidades próprias para o uso dos ambientes de aprendizagem que têm sido os mais utilizados na EAD ou na modalidade de aprendizagem semipresencial.

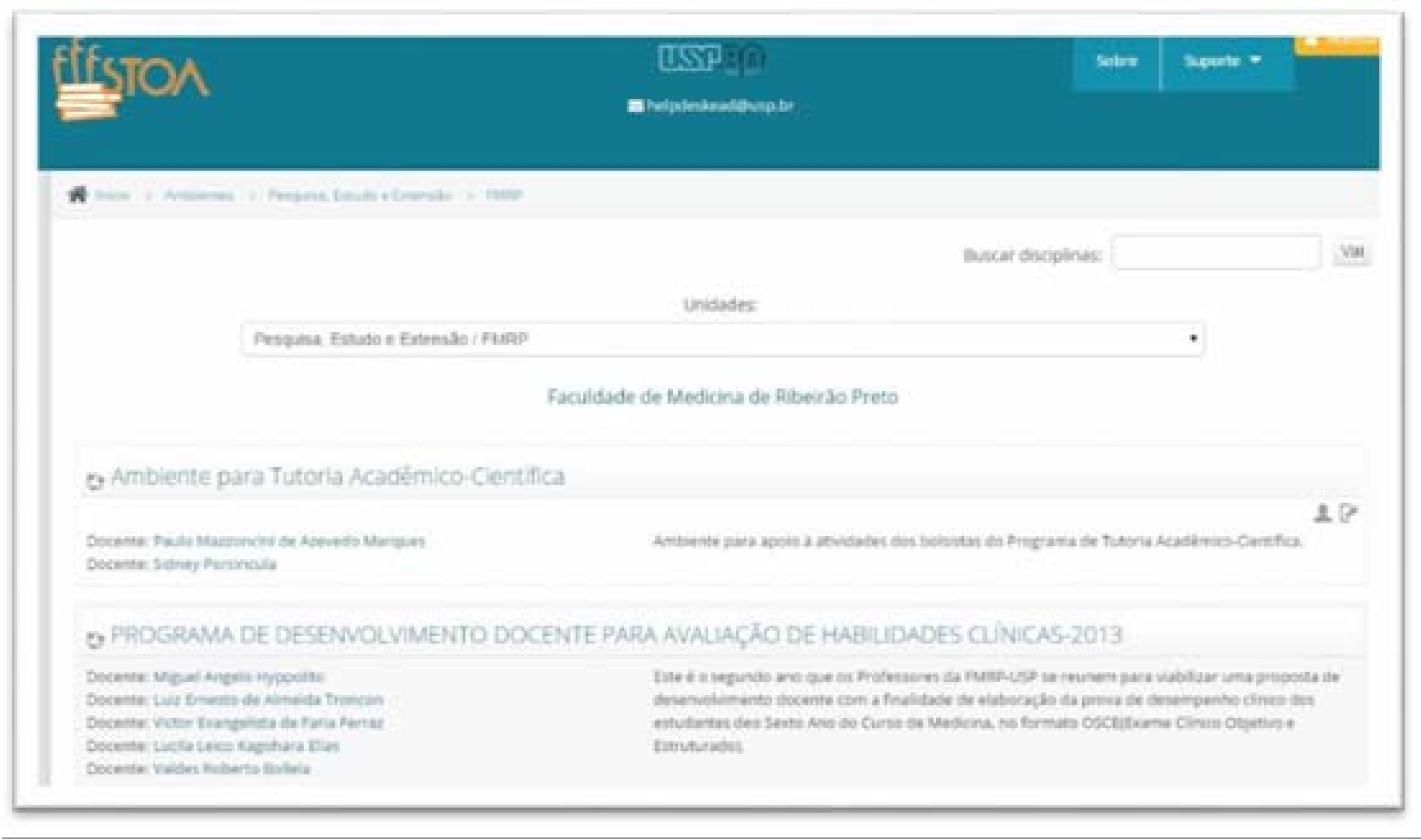

Figura 3 - AVA para atividades de Cultura e Extensão da FMRP. 
- Manejar ferramentas tecnológicas que possibilitem atuar como orientador e facilitador, apoiando o papel do aluno de auto gestor do seu processo de aprendizagem.

- Dominar o uso de metodologias de ensino e de avaliação que sejam centradas na aprendizagem dos estudantes.

- Orientar os estudantes a descobrirem seu ritmo de aprendizagem a fim de conseguirem se organizar para trabalhar de forma independente e a cumprir prazos.

- Trabalhar de forma colaborativa e buscar em equipes multiprofissionais as soluções para as dificuldades que certamente surgem em todo o cenário educacional mediado pelas TICs.

Particularmente no ensino na saúde, a educação mediada pelas TICs passa a ganhar maior força quando as estratégias de comunicação passam a envolver a criação de ambientes e dispositivos que possibilitam um aprendizado mais ativo, tais como simuladores virtuais de casos clínicos e jogos interativos que contribuam para aquisição de algumas habilidades práticas importantes. Nesse ambiente, as avaliações, por sua vez, podem ultrapassar as formas clássicas, podendo ser realizadas em ambientes de simulação realista, possibilitando também a verificação de habilidades e comportamentos específicos esperados. Isso implica, porém, na necessidade de análises constantes das evidências relativas a adequação das condições de aprendizagem (práticas, percepções, formação e engajamento do corpo acadêmico), para se identificar as ferramentas especificas para atender às necessidades do processo de ensino e aprendizagem de cada cenário. Nesse contexto, evidencia-se a necessidade de estruturação de núcleos multiprofissionais como forma de apoio institucional para o estudo, modelagem e implantação de estratégias e de programas de capacitação docente para uma utilização mais efetiva das TICs no ensino na saúde.

\section{Principais pontos de interesse}

- Tecnologias da Informação e Comunicação (TICs) refere-se ao conjunto de tecnologias que permitem o acesso à informação através do uso de telecomunicação.
- Na área da atenção à saúde as TICs têm se tornado instrumentos importantes de apoio às atividades de assistência e de formação profissional.

- Educação a distância (EAD) é a modalidade educacional na qual alunos e professores estão separados, física ou temporalmente e, por isso, faz- se necessária a utilização de meios e tecnologias de informação e comunicação.

- Os recursos tecnológicos disponíveis para o ensino são bastante diversificados, destacando-se alguns como fórum, bibliotecas virtuais, mural de avisos, bate-papo e webconferência; em geral integrados dentro de um Ambiente Virtual de Aprendizagem (AVA), como o moodle, por exemplo.

- Iniciativas governamentais importantes estão atualmente disponíveis para a formação profissional e atenção à saúde com suporte das TICs, destacando-se a Rede Universitária de Telemedicina (RUTE), Universidade Aberta do SUS (UNA-SUS) e o Telessaúde Brasil Redes.

- Competências docentes específicas se fazem necessárias para potencializar possíveis sucessos na utilização das TICs, o que implica na necessidade de estruturação de núcleos multiprofissionais como forma de apoio institucional para uma inserção mais efetiva dessas tecnologias no ensino na saúde.

\section{Referências}

1. Rezende DA, Abreu AF. Tecnologia da Informação Aplicada a Sistemas de Informação Empresariais. São Paulo: Atlas, 2000.

2. Hans O, Rizo C, Enkin M, Jadad A. What is e health: a systematic review of published definitions. J Med Internet Res. 2005; Jan-Mar:7(1).

3. Ruiz JG, Mintzer MJ, Leipzig RM. The Impact of E-learning in Medical. Acad Med. 2006; 81: 207-12.

4. Messina LA; Ribeiro Filho JL. Impactos da Rede Universitária de Telemedicina Ações de educação contínua, pesquisa colaborativa, assistência, gestão e avaliação remota Fase I 2006/2009. Rio de Janeiro: E-papers Serviços Editoriais, 2013.

5. Ribeiro Filho JL. As 100 primeiras unidades de Telemedicina no Brasil e o impacto da Rede Universitária de Telemedicina (RUTE). In Ribeiro Filho JL, Messina LA, Lopes PRL, editors. 1th ed. Rio de Janeiro: E-papers Serviços Editoriais, 2014.

6. Wen CL. Impacto da Rede Universitária de Telemedicina Faculdade de Medicina da Universidade de São Paulo (FMUSP). In: Messina LA, Ribeiro Filho JL, editors. Impactos da Rede Universitária de Telemedicina: Ações de educação contínua, pesquisa colaborativa, assistência, gestão e avaliação remota. 1th ed. Rio de Janeiro: E-papers Serviços Editoriais, 2013. 
7. Silva AB, Moraes IHS. O caso da Rede Universitária de Telemedicina: análise da entrada da telessaúde na agenda política brasileira. Physis, Rio de Janeiro, 2012; 22:3.

8. Sabattini RME, Cardoso SH. O Setor de saúde e a EAD In: Litto FM; Formiga M, editors. Educação a distância: o estado da arte. Volume 2. 1th ed. São Paulo: Pearson Education, 2009. p. 39-46.

9. Alves J. A história da EAD no Brasil, In: Litto FM, Formiga M, editors. Educação a distância: o estado da arte. Volume 1. 1th ed. São Paulo: Pearson Education do Brasil, 2009; p. 9-13.

10. Damásio MM. Educação Presencial X Educação a Distância: reflexões e considerações. Te em Revista. Brasília, 2008; 2 : 75-103.
11. Vianney J. A ameaça de um modelo único para a EaD no Brasil. Colabor@ - Revista Digital da CVA-RICESU, 2008; 5: 17.

12. Cozin LF, Porcíncula S, Suzuki KMF, Azevedo-Marques PM. Transmissão e Gravação em Tempo Real e em Alta Definição com o uso do DVTS na demonstração de Procedimentos de Acesso Cirúrgico realizados em Evento de Neurocirurgia. Revista Brasileira de Inovação Tecnológica em Saúde, 2011: 2: 1-14. 